\title{
Detectable IL-8 and IL-10 in Bronchoalveolar Lavage Fluid from Preterm Infants Ventilated for Respiratory Distress Syndrome
}

\author{
MICHAEL W. BERESFORD AND NIGEL J. SHAW \\ Neonatal Unit, Liverpool Women's Hospital, Liverpool, United Kingdom
}

\begin{abstract}
Pro-inflammatory cytokines such as IL-8 play an important role in the inflammatory response to neonatal airway injury. Difficulty in detecting counter-regulatory cytokines such as IL-10 in lavage fluid from preterm infants led to the suggestion that its deficit may be a factor in the etiology of chronic lung disease of prematurity (CLD). The aim of the study was to determine IL- 8 and IL-10 concentrations in lavage fluid from preterm infants ventilated for respiratory distress syndrome. Fifty infants $<30$ wk gestation were studied who had been randomized to receive a natural or synthetic surfactant. Lavage samples were collected daily for the first week and twice weekly thereafter. Samples were immediately centrifuged and stored at $-70^{\circ} \mathrm{C}$. Cytokine concentrations were quantified in duplicate using commercially available sandwich ELISA kits. Lavage IL-10 concentration, at a minimum initially, rose significant over the first five postnatal days $(p=0.009)$. In the same samples, lavage IL-8 concentrations rose significantly over the first postnatal week $(p<0.001)$, the rise preceding that of IL-10. Infants dying or developing CLD had a significant early rise in both cytokine concentrations. Compared with infants developing CLD, lavage IL-10 concen-
\end{abstract}

ABSTRACT

trations were significantly higher on $\mathrm{d} 1$ among those not developing CLD but significantly lower on $\mathrm{d} 4(p<0.05)$. To conclude, IL-10 is detectable in lavage fluid from ventilated preterm infants and its concentrations rise significantly over the first five postnatal days. In the same samples, IL-8 concentration also rises and this increase precedes the rise in IL-10. (Pediatr Res 52: 973-978, 2002)

$\quad$ Abbreviations
APH, antepartum hemorrhage
BAL, bronchoalveolar lavage
CLD, chronic lung disease of prematurity
HRP, horseradish peroxidase
IUGR, intrauterine growth retardation
IVH, intraventricular hemorrhage
IQR, interquartile range
NB-BAL, nonbronchoscopic BAL
PIH, pregnancy-induced hypertension
RDS, respiratory distress syndrome
TMB, 3,3',5,5'-tetramethylbenzidine

Three studies have previously reported conflicting data on lung lavage IL-10 concentrations in preterm infants with RDS $(3,9,10)$. Initially, Jones et al. (3) suggested that IL-10 mRNA and IL-10 itself was undetectable in most BAL samples collected over the first 96 postnatal hours from preterm infants ventilated for RDS. This apparent absence of IL-10 in lung lavage fluid led to the suggestion that its deficit may be a factor in the etiology of CLD. Using more proximal tracheal aspiration methods of lung lavage, IL-10 was found to be detectable, albeit at low concentrations, when measured over the first 10 postnatal days (9). Recently, IL-10 has been reported to be detectable in lavage fluid obtained using a distal, NB-BAL technique, but in only a minority of infants (10).

The aim of this study was to determine whether IL-10 is detectable over the first two postnatal weeks in bronchoalveolar lavage fluid of preterm infants ventilated for RDS. In addition, this study aimed to measure a pro-inflammatory chemokine (IL-8) in the same samples to assess the relative change over time of these opposing cytokines.
Received February 8, 2002; accepted July 1, 2002.

Correspondence: Michael W Beresford, M.D., Neonatal Unit, Liverpool Women's Hospital, Liverpool L8 7SS, U.K.; e-mail: m_beresford@yahoo.com.

Supported, in part, by the Newborn Appeal, Serono Laboratories, and Britannia Pharmaceuticals.

DOI: 10.1203/01.PDR.0000036626.67304.65 


\section{METHODS}

The study took place at the Liverpool Women's Hospital, U.K., between May 1998 and October 1999.

Subjects. Infants were eligible for entry into the study if they were born at $<30 \mathrm{wk}$ gestation, intubated for presumed surfactant deficiency, and had received exogenous surfactant. Infants were deemed surfactant deficient if, in the opinion of the attending clinician, they had clinical signs of respiratory distress requiring ventilation. Infants were excluded if they had a congenital malformation likely to affect mortality or respiratory outcome. All infants received at least one dose of exogenous surfactant (and a maximum of two) and participated in a randomized controlled trial comparing pumactant to poractant alfa surfactants (11). Consecutive infants randomized in Liverpool to the trial were studied. No infant received corticosteroids after birth during the first postnatal week. All infants were ventilated using conventional mandatory ventilation according to established unit guidelines.

Approval was granted by the local pediatric research ethics committee and written informed parental consent obtained before entry to the study. Demographic and outcome data were collected prospectively. Reason for delivery among infants delivered by cesarean section was defined as the primary obstetric diagnosis appearing in the maternal notes warranting intervention. These included IUGR, defined as fetal growth 2 SD below the mean for gestational age; fetal distress, diagnosed on the basis of cardiotocographic and fetal blood gas monitoring; and suspected chorioamnionitis, diagnosed on the basis of maternal pyrexia, rising white cell count and C-reactive protein concentration, and uterine tenderness. Clinical outcomes included CLD, defined as dependency on supplemental oxygen at $28 \mathrm{~d}$ and at 36 postmenstrual weeks; pneumothorax, defined as intrathoracic, extrapulmonary air leak requiring insertion of chest drain; pulmonary hemorrhage, defined as spontaneous appearance of blood or blood-stained fluid in the endotracheal tube; severe IVH, defined as IVH with ventricular enlargement or parenchymal hemorrhagic lesions.

Protocol for BAL and sample processing. NB-BAL was performed using a standardized technique at the time of routine suctioning. Infants were positioned supine with the head turned toward the left. Cardiorespiratory monitoring continued throughout the procedure. Inspired oxygen concentration was increased just before performing BAL to maintain oxygen saturations $>90 \%$ throughout the lavage procedure. The endotracheal tube was disconnected from the ventilator and an end-hole FG 6 suction catheter carefully inserted until slight resistance felt. A $1.0 \mathrm{~mL} / \mathrm{kg}$ (up to a maximum volume of 1.0 $\mathrm{mL}$ ) aliquot of normal saline was instilled gently via the suction catheter, followed by a $0.5 \mathrm{~mL}$ air bolus to clear the catheter dead space. The catheter was then connected to a suction trap to which $80-100 \mathrm{~mm} \mathrm{Hg}$ of suction pressure was applied while simultaneously withdrawing the catheter from the endotracheal tube. The infant was reconnected to the ventilator and monitored to ensure a stable state. The whole process was then repeated using a second identical volume aliquot of normal saline. The two aliquots were pooled and centrifuged at $2000 \mathrm{rpm}$ for 5 min using a Heraeus Sepatech
Biofuge-15 centrifuge (Germany). Cell-free supernatant was aliquotted into polypropylene microtubes and frozen at $-70^{\circ} \mathrm{C}$ within $15 \mathrm{~min}$ of collection. Samples were thawed once, immediately before analysis. Day one samples were collected within the first $24 \mathrm{~h}$ after delivery, at least $6 \mathrm{~h}$ after the last surfactant administration. Samples were collected on a daily basis for the first week and twice a week thereafter until extubation.

Cytokine assays. Single batch, double-antibody sandwich, high-sensitivity ELISA kits were used to quantify both cytokines following manufacturer's protocols (R\&D Systems Europe Ltd., Abingdon, U.K.). In brief, quantification of IL-8 took place using mouse monoclonal anti-human IL-8 antibody (MAB208, R\&D Systems) diluted to $4.0 \mu \mathrm{g} / \mathrm{mL}$ in PBS. Standards used were recombinant human IL-8 (208-IL-010, R\&D Systems), serially diluted from $5000 \mathrm{pg} / \mathrm{mL}$. Lavage samples were diluted $1: 20$ with $0.1 \%$ BSA, $0.05 \%$ Tween 20 in PBS while on ice, immediately before use. Secondary detection antibody was biotinylated goat anti-human IL-8 antibody (BAF208, R\&D Systems) diluted using 0.1\% BSA, 0.05\% Tween20 in PBS and plated at a concentration of $25 \mathrm{ng} / \mathrm{mL}$.

Quantification of IL-10 took place using mouse monoclonal anti-human IL-10 capture antibody (MAB217, R\&D Systems) diluted in PBS to $2.5 \mu \mathrm{g} / \mathrm{mL}$. Standards used were recombinant human IL-10 (217-IL-005, R\&D Systems) serially diluted in $0.1 \%$ BSA in PBS from $5000 \mathrm{pg} / \mathrm{mL}$. Lavage samples were diluted $1: 4$ with $0.1 \%$ BSA, $0.05 \%$ Tween 20 in PBS while on ice, immediately before use. Secondary detection antibody was biotinylated goat anti-human IL-10 antibody (BAF217, R\&D Systems) diluted using 0.1\% BSA, 0.05\% Tween 20 in PBS and plated at a concentration of $500 \mathrm{ng} / \mathrm{mL}$.

All reagents were added in $100 \mu \mathrm{L}$ volumes per well, apart from blocking buffers. Incubation of capture antibodies took place overnight at room temperature. Blocking took place for $90 \mathrm{~min}$ at room temperature using $300 \mu \mathrm{L}$ per well blocking buffer (1\% BSA, $5 \%$ sucrose, $0.05 \%$ sodium azide in PBS). Incubation of samples and standards, and then of the secondary antibody, took place after covering the plate with an adhesive strip on a plate agitator for $1 \mathrm{~h}$ at $37^{\circ} \mathrm{C}$. Between each stage, all wells were aspirated, washed forcefully five times with wash buffer $(0.05 \%$ Tween 20 in PBS), and blotted dry. For both ELISA, dilution of streptavidin-HRP conjugate (43-4323, 1.25 $\mathrm{mg} / \mathrm{mL}$, Zymed, Cambridge Bioscience, U.K.) was performed using $0.1 \%$ BSA, $0.05 \%$ Tween 20 in PBS, at 1:20,000 for IL-8 and 1:4,000 for IL-10. Incubation took place at room temperature for $20 \mathrm{~min}$. Incubation with TMB/peroxide took place in the dark at room temperature for $20 \mathrm{~min}$ before stopping the reaction using $2 \mathrm{~N} \mathrm{H}_{2} \mathrm{SO}_{4}$. Measurement of absorbance took place using wavelengths of 450 and $550 \mathrm{~nm}$ and standard curves were constructed from which samples were read.

All samples were analyzed in duplicate. Cytokine concentrations are expressed per volume of lavage fluid $(\mathrm{pg} / \mathrm{mL})$. Intra-assay coefficient of variance for IL-8 and IL-10 was $6.9 \%$ and $5.3 \%$, respectively. Interassay coefficient of variance was $11.1 \%$ and $12.3 \%$, respectively. Coefficient of repeatability for 672 paired measures of IL-8 was $16.8 \%$ and for 255 paired measures of IL-10 was $11.4 \%$. 
Data analysis. Statistics were performed using the SPSS 10.0 for Windows statistical package (SPSS, Chicago, IL). Unless otherwise stated, numerical results are presented as median (IQR). Test of normality of distribution took place using the one-sample Kolmogorov-Smirnov test. Two-tailed tests were used for all statistical comparisons. Statistical significance was defined as a $p$ value of $<0.05$.

Comparisons between groups took place using the MannWhitney $U$ test for continuous data and the $\chi^{2}$ or Fisher's exact test for categorical data. Paired data were analyzed using the Wilcoxon signed-rank test. Correlations between continuous data used Spearman's $\rho$ correlation coefficient. Assessment of duplicate measures used the method described by Bland and Altman (12). The coefficient of variation was calculated using Arcus Quickstat 1.0 statistical package (Research Solutions, Cambridge, U.K.). Repeated-measures ANOVA was used to analyze measurements of surfactant protein concentration for the same infant over time. Natural logarithmic transformation of nonparametric data took place where necessary to allow these analyses to be carried out (resulting in normally distributed data). Data points below the level of detection of the assays were not included in the analyses.

\section{RESULTS}

Fifty infants were enrolled to this study from the first 65 (77\%) live-born infants randomized to the surfactant trial at Liverpool Women's Hospital. Reasons for noninclusion were as follows: randomized but not requiring intubation (2); extubation occurring before the initial sampling window (5); routine suctioning not performed on d 1 due to pulmonary hemorrhage or severity of condition (3) or postnatal death (2); operational reasons (3). Median (IQR) time after delivery to initial surfactant dose administration was $7 \mathrm{~min}$ (5-12 min). Forty-nine (98\%) infants received a second surfactant dose after $12 \mathrm{~h}$. All mothers had received at least one dose of antenatal steroids, 46 (92\%) receiving two or more.

Table 1 shows summary demographic and outcome data for

Table 1. Summary of demographic and outcome data for study infants

\begin{tabular}{lc}
\hline Demographic/outcome variable & $\begin{array}{c}\text { Study cohort }(n=50 \text { unless } \\
\text { otherwise stated) }\end{array}$ \\
\hline Gestation (wk)* & $27.5(1.5)$ \\
Birth weight (g)* & $914(278)$ \\
Mortality (\%) & \\
Neonatal & $9(18)$ \\
Predischarge & $15(30)$ \\
CLD at 36 wk (\%) & \\
All infants & $24(48)$ \\
Survivors at 36 wk & $24 / 40(60)$ \\
CLD or death at 36 wk (\%) & $34(68)$ \\
Among survivors to discharge & \\
Total ventilated days** & $6(3-15)$ \\
Total hospital days** & $81(56-107)$ \\
Home oxygen (\%) & $15 / 35(43)$ \\
All infants & \\
Pneumothorax (\%) & $6(12)$ \\
Pulmonary hemorrhage (\%) & $2(4)$ \\
Severe IVH (\%) & $5(10)$ \\
\hline
\end{tabular}

* Mean (standard deviation); ** median (IQR). the whole cohort. Twenty (40\%) infants were born after spontaneous vaginal delivery, six (12\%) by semielective cesarean section for IUGR, and three (6\%) by assisted delivery. Of the $21(42 \%)$ infants born by emergency cesarean section, 10 $(20 \%)$ were because of pregnancy-induced hypertension or antepartum hemorrhage, six (12\%) for fetal distress, and five $(10 \%)$ due to suspected chorioamnionitis. Sixteen $(32 \%)$ of the infants were from twin deliveries. Demographic and clinical outcomes between surfactant groups in this study were similar to the overall trial findings (11).

From the whole cohort, 15 infants died before discharge. Nine of these were due to a primary respiratory cause, of which eight had received pumactant. Fewer infants died before discharge home if they had been treated with poractant alfa (4/25 versus 11/25), in keeping with the overall trial findings (11). Compared with those discharged home, infants dying were significantly lighter at birth [734 g (600-917 g) versus $920 \mathrm{~g}$ (724-1070 g), $p=0.02]$, with higher Clinical Risk Index for Babies (CRIB) scores [8.5 (6-13) versus $4(2-8), p=0.001]$. There was no significant difference in gender or gestational age between these groups. Those infants alive at 36 postmenstrual weeks with CLD had been born lighter [804 g (648-993 g) versus $1005 \mathrm{~g}(858-1257 \mathrm{~g}), p=0.009]$, at lower gestational age [26 wk (25-28) versus $28 \mathrm{wk}(27-29), p=0.008)$, with higher CRIB scores [6 (3-8) versus $2.5(2-6), p=0.031]$ compared with those without CLD. There was no significant difference in birth-weight or gestational age between those alive with CLD at 28 postnatal days and those without.

Median (IQR) percentage volume return of BAL from all 50 infants was $50 \%(42-58 \%)$ and supernatant volume was $93 \%$ (91-96\%). From 50 infants, 276 BAL samples were analyzed for IL-8 concentration and 264 of the same samples were analyzed for IL-10. Median BAL IL-8 concentration from all samples was $13,121 \mathrm{pg} / \mathrm{mL}(5,983-24,426 \mathrm{pg} / \mathrm{mL})$ and $\mathrm{BAL}$ IL-10 concentration $570 \mathrm{pg} / \mathrm{mL}(210-906 \mathrm{pg} / \mathrm{mL})$. IL-10 was detectable in $89 \%$ of samples analyzed.

Over the first postnatal week, neither BAL IL-8 nor IL-10 concentration correlated significantly with gestational age. Birth weight correlated significantly with BAL IL-10 concentration only on $\mathrm{d} 2(r=-0.367, p=0.02)$ and at no time with BAL IL-8 concentration. Infants born with suspected chorioamnionitis had significantly higher BAL IL-8 concentrations on $\mathrm{d} 1$ compared with infants without $[12,025 \mathrm{pg} / \mathrm{mL}(11,962$ $12,509 \mathrm{pg} / \mathrm{mL})$ versus $4171 \mathrm{pg} / \mathrm{mL}(1943-8929 \mathrm{pg} / \mathrm{mL}), p=$ 0.03 ] but at no other time point. There was no difference in BAL IL-10 concentrations between these groups of infants. Infants delivered because of growth retardation had significantly lower BAL IL-10 concentrations on $\mathrm{d} 2$ compared with those without $[131 \mathrm{pg} / \mathrm{mL}(38-334 \mathrm{pg} / \mathrm{mL})$ versus $611 \mathrm{pg} / \mathrm{mL}$ (455-995 $\mathrm{pg} / \mathrm{mL}), p=0.01]$ but at no other time point. However, BAL IL-8 concentrations did not differ significantly at any time point between these infants. There was no significant difference in BAL IL-8 or IL-10 concentrations between infants born by emergency cesarean section and those not or born with fetal distress and those without. There was no significant correlation among survivors between BAL IL-8 or IL-10 concentration and duration of ventilation or length of hospital stay. 
Figure 1 shows BAL IL-8 and Figure 2 shows BAL IL-10 concentrations for the whole study cohort over the first two postnatal weeks. Median IL-8 concentration was at a minimum on the first postnatal day. BAL IL-8 concentrations rose significantly over the first four postnatal days $(p<0.001)$ and continued to rise until the end of the first postnatal week $(p<$ 0.001). Although the median BAL IL-8 concentration falls away in the second postnatal week, this change was not statistically significant. Paired samples were significantly different from $\mathrm{d} 1$ concentrations at each time point over the first two postnatal weeks $(p<0.01)$.

In contrast, there was no significant rise in BAL IL-10 concentration for the whole cohort over the first $4 \mathrm{~d}$. IL-10 concentration was at a minimum over the first three postnatal days but then rose. There was a significant rise in IL-10 concentration over the first five postnatal days $(p=0.009)$. There was a nonsignificant trend toward IL-10 concentrations falling away after this. Paired samples were significantly different from d 1 concentrations on $\mathrm{d} 4$ and 5 ( $p=0.05$ and $p=$ 0.01 , respectively).

Over the first postnatal week, there was no statistically significant difference in BAL IL-8 concentrations between infants treated with pumactant or poractant alfa. Infants given poractant alfa had a higher median BAL IL-8 concentration in the second postnatal week, and this difference was statistically significant on d 8-10 [poractant alfa: $27,213 \mathrm{pg} / \mathrm{mL}(23,292-$ $34,101 \mathrm{pg} / \mathrm{mL})$ versus pumactant: $14,975 \mathrm{pg} / \mathrm{mL}(8,551-$ $19,460), p=0.03]$. BAL IL-10 concentration varied between surfactant groups, being lower in poractant alfa-treated infants over the first $4 \mathrm{~d}$ and greater in the latter half of the first week. However, these differences were not statistically significantly.

There was no significant difference over the first two postnatal weeks in BAL IL-8 or IL-10 concentrations between infants dying before discharge and those discharged home. BAL IL-8 concentrations rose significantly over the first $4 \mathrm{~d}$ in those dying $(p=0.009)$ and those surviving $(p=0.004)$ and over the first week ( $p=0.002$ and $p=0.02$, respectively). Unlike infants surviving, infants dying showed a significant

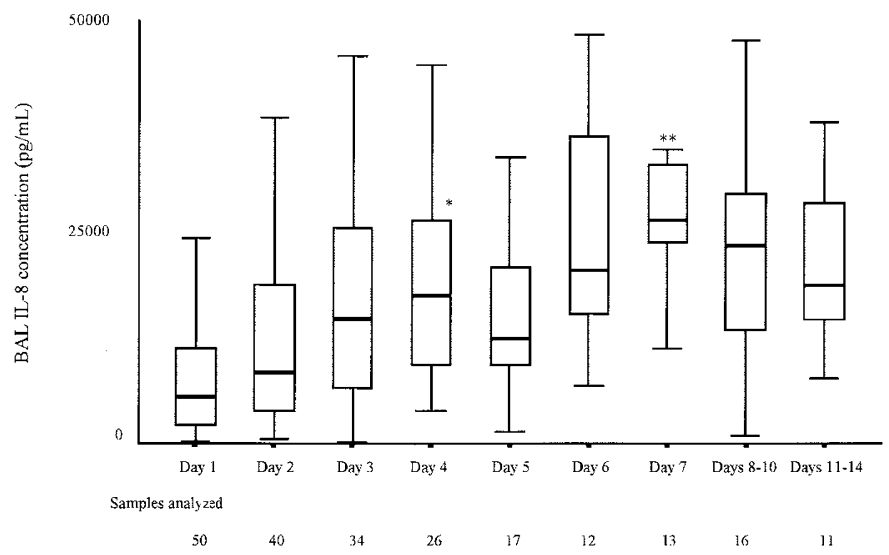

Figure 1. Box-and-whisker plot of BAL IL-8 concentrations for whole cohort over the first two postnatal weeks. BAL IL-8 concentrations rose significantly over the first $4 \mathrm{~d}(* p<0.001)$ and first postnatal week $(* * p<$ $0.001)$. Paired samples were significantly different from d 1 concentrations at each time point over the first two postnatal weeks $(p<0.01)$.

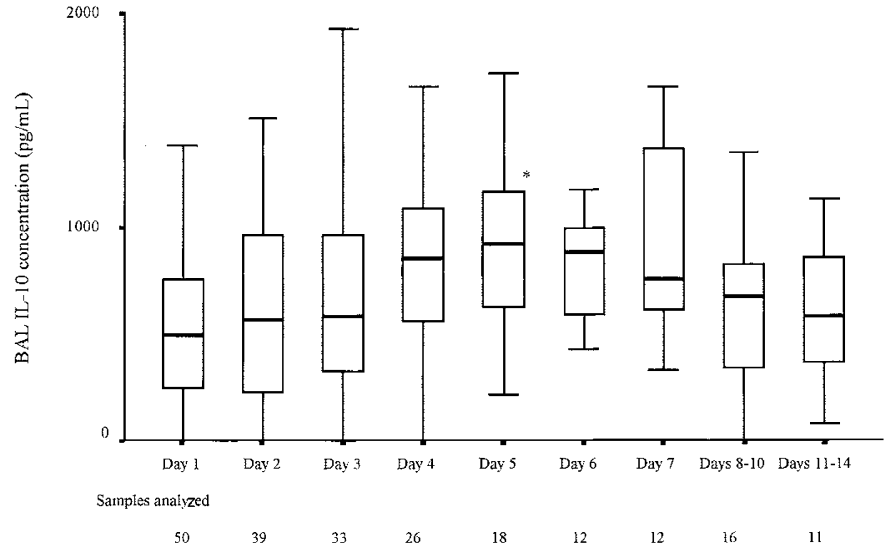

Figure 2. Box-and-whisker plot of BAL IL-10 concentrations for whole cohort over the first two postnatal weeks. BAL IL-10 concentrations were at a minimum over the first three postnatal days before rising significantly by $\mathrm{d} 5$ $\left({ }^{*} p=0.009\right)$.

rise in BAL IL-10 concentration over the first four postnatal days $(p=0.04)$.

BAL IL-8 concentration did not differ significantly over the first four postnatal days between survivors developing CLD and those not developing CLD. During this period, BAL IL-8 concentrations rose significantly in those developing $\operatorname{CLD}(p=$ 0.001). Infants not developing CLD by d 28 had significantly higher BAL IL-10 concentrations on d 1 compared with those not developing CLD $(p=0.03)$. Concentrations fell and were significantly lower on $\mathrm{d} 4$ compared with infants developing CLD ( $p=0.02$, Fig. 3). BAL IL-10 concentrations rose significantly over the first five postnatal days $(p=0.003)$ among survivors who developed CLD. Although similar trends were present, differences in BAL IL-10 concentration between those with and without CLD at 36 postmenstrual weeks were

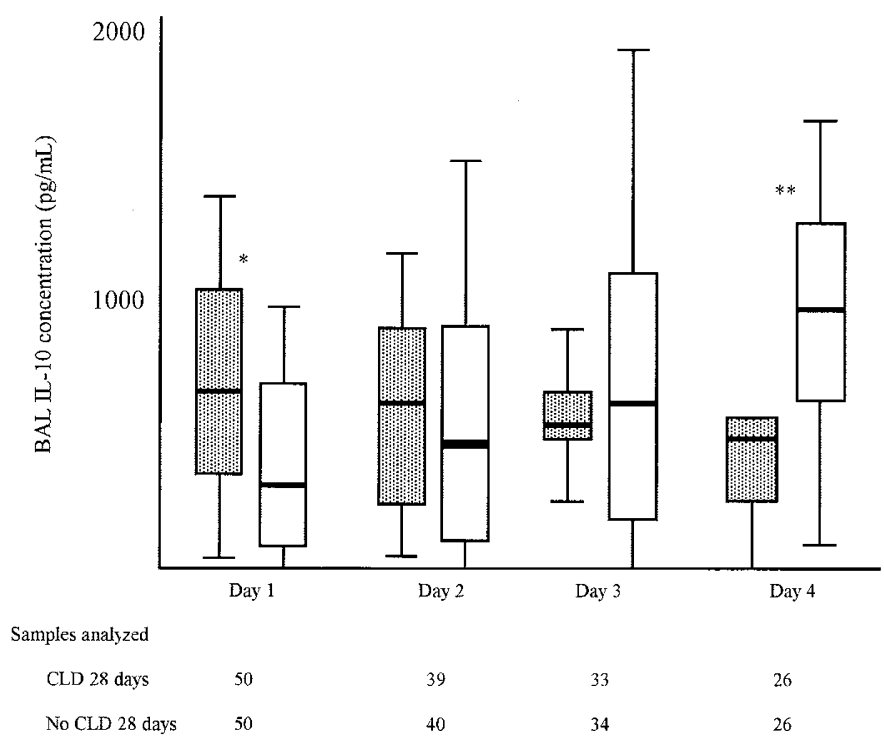

Figure 3. Box-and-whisker plot of BAL IL-10 concentrations among survivors with (shaded boxes) and without (open boxes) CLD at d 28. BAL IL-10 concentrations were significantly higher on d 1 among survivors not developing CLD by $\mathrm{d} 28$ in contrast to those with CLD $\left({ }^{*} p=0.03\right)$. On $\mathrm{d} 4$, BAL IL-10 concentrations were significantly lower in survivors without CLD on d 28 compared with those with CLD $(* * p=0.02)$. 
not statistically significant. Infants developing CLD or dying by $36 \mathrm{wk}$ had significantly higher BAL IL- 8 concentrations on d 4 when compared with those recovering $[20,935 \mathrm{pg} / \mathrm{mL}$ $(11,956-27,407 \mathrm{pg} / \mathrm{mL})$ versus $7,432 \mathrm{pg} / \mathrm{mL}(5,537-11,964$ $\mathrm{pg} / \mathrm{mL}) ; p=0.04]$. Group comparisons between survivors developing CLD and those not developing CLD were not possible after $\mathrm{d} 4$, as only a maximum of three infants in the latter group were intubated at any time point thereafter.

\section{DISCUSSION}

This study has demonstrated for the first time the pattern of change over the first two postnatal weeks of a pro- and an anti-inflammatory cytokine measured in the same samples from ventilated preterm infants with RDS.

We have demonstrated that, using a standardized NB-BAL technique and a commercially available ELISA kit, IL-10 is detectable in BAL fluid from a very preterm population. Two studies have previously explored BAL IL-10 concentrations in preterm infants using NB-BAL $(3,10)$. Jones et al. (3) found that BAL IL-10 was immeasurable in most samples collected from 17 preterm infants over the first four postnatal days and that IL-10 mRNA was not detectable in samples analyzed from five preterm infants. Jónsson et al. (10) reported IL-10 detectable in $12 / 46(26 \%)$ preterm infants studied. There are a number of possible reasons why IL-10 was detected more readily in our study. Although broadly similar, differences in sample collection and processing techniques may have altered measurability of IL-10. Using $0.5-\mathrm{mL}$ aliquots of saline for each lavage, Jónsson et al. (10) flushed the suction catheter with an equal volume of saline after aspiration. Dilution of samples preanalysis could have reduced detectability of IL-10. Factors within BAL fluid may affect immunologic availability for binding to the ELISA antibodies (3). Differences in sensitivity/specificity of the ELISA may alter measured IL-10 concentration, and differences in the manufacture of antibodies/ standards are not necessarily provided for (3). Cytokine concentrations vary between and within infants over time. Our study demonstrates BAL IL-10 concentrations are lowest over the first three postnatal days. In the study by Jones et al. (3), sampling only over the initial 96 postnatal hours may have reduced their chance of detecting IL-10. They speculate that maturational differences with increasing gestational age may influence IL-10 expression. Our data, however, showed that BAL IL-10 concentration did not correlate with gestational age at any time over the first postnatal week and correlated negatively with birth weight only on $\mathrm{d} 2$. Furthermore, median gestational age was lower in our study than that of Jones et al. (3). Growth-retarded infants did, however, have lower BAL IL-10 concentrations on $\mathrm{d} 2$. Understanding the mechanisms controlling IL-10 expression in the preterm infants and how this may vary with development is still poorly understood (13). Differences in population characteristics, antenatal steroid uptake, and surfactant usage may also explain differences from our study $(3,10)$. In contrast, using tracheal aspiration methodology, McColm et al. (9) showed that $56 \%$ of samples from 17 preterm infants had detectable IL-10 when collected over the first 10 postnatal days. Differences in detection rate and concentration of BAL IL-10 between this study and ours may be due partly to more proximal sampling of the bronchial tree using tracheal aspiration compared with NB-BAL (14). Interest in distal alveolar mechanisms of disease meant that a NB-BAL technique was adopted for this study following internationally recognized methodology (15).

In agreement with previous reports, BAL IL-8 concentrations for the whole cohort rose significantly over the first $4 \mathrm{~d}$ $(4,16-18)$. As in our study, no data has reported NB-BAL IL-8 concentrations to be significantly different between infants going on to develop CLD and those who do not, when measured over the first four postnatal days (16-18). Previous reports indicate that infants with CLD may have significantly higher BAL IL-8 concentrations by the end of the first postnatal week compared with those without CLD. Paucity of sampling after d 4 among infants recovering without CLD makes comparisons after this time inappropriate in our study. In contrast to infants not developing CLD, survivors developing CLD had a significant increase in BAL IL-8 concentrations over the first $4 \mathrm{~d}$. Although other NB-BAL studies indicate an early rise in IL-8 concentration among infants developing CLD, none report whether the change is statistically significant (3, 4, 16-18). In contrast to infants not developing CLD, infants developing CLD and those who subsequently died had a significant rise in BAL IL-10 over the first four postnatal days. Despite a poor clinical outcome, these infants had a marked counter-regulatory response to injury. This is in contrast to the hypotheses that an apparent lack of BAL IL-10 may result in poor prognosis (3). As well as infants dying, those surviving also had significant rise in BAL IL-8 concentration over the initial postnatal period and there was no significant difference in BAL IL-8 or IL-10 concentration between these outcomes. However, sample size was not calculated to detect significant differences between outcome groups. There was no published NB-BAL data available from this very preterm population treated with poractant alfa and pumactant before commencing this study, so formal sample size determination was not possible. A sample size of 50 was chosen pragmatically and prospectively to be among the largest study in this field. Indeed, it is the largest study to date of preterm infants in which BAL IL-10 has determined $(3,9,10)$. However, from our data, we have shown that infants recovering from RDS, or going on to develop CLD or even die, have both a marked pro-inflammatory and counter-regulatory cytokine response. Multiple factors, including cytokine response, will influence clinical outcome but timing as well as magnitude of response may be critical to this. Modulation of the inflammatory response with treatment agents may alter outcome. We have shown for the whole cohort that a delayed but significant rise takes place in BAL IL-10 concentration. The rise in IL-10 appears to follow from the initial rise in BAL IL-8 concentration. IL-10 helps regulate the inflammatory response and a temporal relationship between the two cytokines in the inflammatory cascade appears to be demonstrated here.

Few studies investigating BAL cytokine concentrations using NB-BAL methodology state the type of surfactant therapy used (16-20) and only one reports comparative (cellular) data between surfactant-treated infants and controls (19). The effect 
of surfactants on inflammatory mediators obtained using NBBAL is therefore unknown. This is the first study comparing BAL IL-8 and IL-10 concentrations in infants randomly assigned to two different types of surfactant therapy. Neither of the two surfactant proteins in poractant alfa is known to have effects on the immunologic defense mechanisms of the airways (21). BAL IL-8 and IL-10 concentrations did not differ significantly over the first week between infants treated with pumactant or poractant alfa, despite physiologic differences in the effects of these surfactants $(22,23)$ and marked variation in clinical outcome (11). Sample size may have limited the power of this study to detect significant differences in BAL cytokine concentrations between surfactants.

No universally accepted method has been established to standardize for solute dilutions in BAL samples but techniques include estimation of urea dilution and lavage secretory component of IgA concentration $(24,25)$. Recent consensus statements advise that until such an appropriate marker is determined, results should be expressed as concentration per milliliter of BAL fluid $(15,26)$. This facilitated comparison of our data with published reports of BAL IL-10 that used the same units $(3,9,10)$. Percentage BAL fluid return was comparable to recommended values $(15,24)$.

An inherent limitation of using BAL as an investigative technique is obtaining samples from extubated infants recovering from RDS. Consequently, reports may include older (term) infants in control populations that can significantly affect interpretation of data $(18,27,28)$. Few survivors in our study not developing CLD remained intubated after $\mathrm{d} 4$. The effect of prematurity on lung defense mechanisms remains poorly understood (13). To contrast potential differences between disease states, only infants born at $<30 \mathrm{wk}$ gestation were studied.

Neither IL-8 or IL-10 mRNA concentrations, nor cytokine concentrations in the cellular fraction of BAL fluid were measured in these infants. Detection, quantification, and analysis of change and association of a given cytokine within BAL fluid do not necessarily translate into physiologic effect. Measurement of mRNA and cellular cytokine concentrations in future studies would further contribute to understanding the role of these important cytokines in preterm respiratory disorders.

In conclusion, IL-10 is detectable in BAL fluid from preterm infants ventilated for RDS and concentrations rise significantly over the first five postnatal days. In the same samples, IL- 8 concentration also rises and this increase precedes the rise in IL-10.

\section{REFERENCES}

1. Groneck P, Speer CP 1995 Inflammatory mediators and bronchopulmonary dysplasia. Arch Dis Child Fetal Neonatal Ed 73:F1-F3
2. Kotecha S 1996 Cytokines in chronic lung disease of prematurity. Eur J Pediatr 155(suppl 2):S14-S17

3. Jones CA, Cayabyab RG, Kwong KY, Stotts C, Wong B, Hamdan H, Minoo P, Delemos RA 1996 Undetectable interleukin (IL)-10 and persistent IL-8 expression early in hyaline membrane disease: a possible developmental basis for the predisposition to chronic lung inflammation in preterm newborns. Pediatr Res 39:966-975

4. Kwong KY, Jones CA, Cayabyab R, Lecart C, Stotts CL, Randhawa I, Ramanathan R, Khuu N, Minoo P, Delemos RA 1998 Differential regulation of IL-8 by IL-1beta and TNFalpha in hyaline membrane disease. J Clin Immunol 18:71-80

5. Tullus K, Noack GW, Burman LG, Nilsson R, Wretlind B, Brauner A 1996 Elevated cytokine levels in tracheobronchial aspirate fluids from ventilator treated neonates with bronchopulmonary dysplasia. Eur J Pediatr 155:112-116

6. Moore KW, O'Garra A, de Waal Malefyt R, Vieira P, Mosmann TR 1993 Interleukin10. Annu Rev Immunol 11:165-190

7. de Waal Malefyt R, Abrams J, Bennett B, Figdor CG, de Vries JE 1991 Interleukin-10 inhibits cytokine synthesis by human monocytes. An autoregulatory of IL-10 produced by monocytes. J Exp Med 174:1209-1220

8. Fiorentino DF, Zlotnik A, Mosmann TR, Howard M, O'Garra A 1991 IL-10 inhibits cytokine production by activated macrophages. J Immunol 147:3815-3822

9. McColm J, Stenson BJ, Biermasz N, McIntosh N 2000 Measurement of interleukin 10 in bronchoalveolar lavage from preterm ventilated infants. Arch Dis Child Fetal Neonatal Ed 82:F156-F159

10. Jónsson B, Li H-Y, Noack G, Brauner A, Tullus K 2000 Downregulatory cytokines in tracheobronchial aspirate fluid from infants with chronic lung disease of prematurity. Acta Paediatr 89:1375-1380

11. Ainsworth SB, Beresford MW, Milligan DWA, Shaw NJ, Matthews JNS, Fenton AC, Ward Platt MP 2000 Pumactant and poractant alfa for treatment of respiratory distress syndrome in neonates born at 25-29 weeks' gestation: a randomised trial. Lancet 355:1387-1392

12. Bland JM, Altman DG 1986 Statistical methods for assessing agreement between two methods of clinical measurement. Lancet 1:307-310

13. Marshall-Clarke S, Reen D, Tasker L, Hassan J 2000 Neonatal immunity: how well has it grown up. Immunol Today 21:35-41

14. Dargaville PA, South M, McDougall PN 1999 Comparison of two methods of diagnostic lung lavage in ventilated infants with lung disease. Am J Respir Crit Care Med 160:771-777

15. ERS Task Force 2000 Bronchoalveolar lavage in children. ERS Task Force on bronchoalveolar lavage in children. Eur Respir J 15:217-231

16. Jónsson B, Tullus K, Brauner A, Lu Y, Noack G 1997 Early increase of TNF alpha and IL-6 in tracheobronchial aspirate fluid indicator of subsequent chronic lung disease in preterm infants. Arch Dis Child Fetal Neonatal Ed 77:F198-F201

17. Little S, Dean T, Bevin S, Hall M, Ashton M, Church M, Warner J, Shute J 1995 Role of elevated plasma soluble ICAM-1 and bronchial lavage fluid IL-8 levels as markers of chronic lung disease in premature infants. Thorax 50:1073-1079

18. Kotecha S, Chan B, Azam N, Silverman M, Shaw RJ 1995 Increase in interleukin-8 and soluble intercellular adhesion molecule-1 in bronchoalveolar lavage fluid from premature infants who develop chronic lung disease. Arch Dis Child Fetal Neonatal Ed 72:F90-F96

19. Arnon S, Grigg J, Silverman M 1993 Pulmonary inflammatory cells in ventilated preterm infants: effect of surfactant treatment. Arch Dis Child 69:44-48

20. Murch SH, Costeloe K, Klein NJ, MacDonald TT 1996 Early production of macrophage inflammatory protein- $1 \alpha$ occurs in respiratory distress syndrome and is associated with poor outcome. Pediatr Res 40:490-497

21. Creuwels LAJM, van Golde LMG, Haagsman HP 1997 The pulmonary surfactant system: biochemical and clinical aspects. Lung 175:1-39

22. Takahashi A, Nemoto T, Fujiwara T 1994 Biophysical properties of protein-free, totally synthetic pulmonary surfactants, ALEC and Exosurf, in comparison with surfactant TA. Acta Paediatr Jpn 36:613-618

23. Corcoran JD, Berggren P, Sun B, Halliday HL, Robertson B, Curstedt T 1994 Comparison of surface properties and physiological effects of a synthetic and a natural surfactant in preterm rabbits. Arch Dis Child Fetal Neonatal Ed 71:F165-F169

24. Kotecha S 1999 Bronchoalveolar lavage of newborn infants. Pediatr Pulmonol Suppl 18:122-124

25. Dargaville PA, South M, Vervaart P, McDougall PN 1999 Validity of markers of dilution in small volume lung lavage. Am J Respir Crit Care Med 160:778-784

26. Haslam PL, Baughman RP 1999 Report of ERS Task Force: guidelines for management of acellular components and standardization of BAL. Eur Respir J 14:245-248

27. Kotecha S, Wangoo A, Silverman M, Shaw RJ 1996 Increase in the concentration of transforming growth factor beta-1 in bronchoalveolar lavage fluid before development of chronic lung disease of prematurity. J Pediatr 128:464-469

28. Kotecha S, Wilson L, Wangoo A, Silverman M, Shaw RJ 1996 Increase in interleukin (IL) $-1 \beta$ and IL-6 in bronchoalveolar lavage fluid obtained from infants with chronic lung disease of prematurity. Pediatr Res 40:250-256 